\title{
Raised serum 25-hydroxyvitamin D levels in patients with active diabetic foot ulcers
}

\author{
Mohsen Afarideh†, Parvaneh Ghanbari†, Sina Noshad, Alireza Ghajar, Manouchehr Nakhjavani and \\ Alireza Esteghamati* \\ Endocrinology and Metabolism Research Center (EMRC), Vali-Asr Hospital, School of Medicine, Tehran University of Medical \\ Sciences, PO Box 13145-784, Tehran, Iran
}

(Submitted 13 August 2015 - Final revision received 22 February 2016 - Accepted 25 February 2016 - First published online 8 April 2016)

\begin{abstract}
Studies have emerged to demonstrate bidirectional changes in circulating cytokines of inflammation in active diabetic foot ulcers (DFU). To further expand the understanding of inflammatory status present in chronic active DFU, we comparatively assessed the associations of selected pro-inflammatory cytokines and 25-hydroxyvitamin D $(25(\mathrm{OH}) \mathrm{D})$ with the presence of DFU. In a cross-sectional setting, thirty patients with type 2 diabetes and active DFU matched with thirty control non-ulcerative patients with type 2 diabetes and twenty-eight healthy subjects underwent anthropometric and biochemical assessment of study parameters. Recruited patients with DFU were selected from the grade II active chronic DFU at the time of hospitalisation according to the University of Texas wound classification system. Patients with DFU and controls had comparable age, sexual distribution, diastolic blood pressure and TAG, LDL-cholesterol and glycated $\mathrm{Hb}$. The trend changes from healthy controls towards DFU showed a significant increase for serum monocyte chemoattractant protein-1, IL-6, 25(OH)D and highly sensitive C-reactive protein and a decrease for IL-8. In the multivariate adjusted logistic regression model, $25(\mathrm{OH}) \mathrm{D}$ emerged as the only independent correlate of DFU (OR 2.194; $95 \%$ CI $1.003,4.415)$. Unprecedented increase of serum $25(\mathrm{OH}) \mathrm{D}$ in chronic active DFU is possibly related to a selective alteration in the inflammatory status. In particular, $25(\mathrm{OH}) \mathrm{D}$ and IL-8 seem to share a common pathway in the pathogenesis of diabetic foot.
\end{abstract}

Key words: Diabetic foot ulcers: Monocyte chemoattractant protein-1: 25-Hydroxyvitamin D: IL-8: IL-6: Highly sensitive C-reactive protein

In 2011, 366 million people had type 2 diabetes, corresponding to $7 \cdot 0 \%$ of the world's population ${ }^{(1)}$. Up to a quarter of this population is being threatened with the lifetime risk of developing diabetic foot ulcers (DFU) ${ }^{(2)}$. Every year more than a million of this subgroup require lower extremity amputations as a consequence, with over 80000 of them being reported in the USA alone ${ }^{(3)}$. Although reports of amputation rate for developing countries are scant, in one simulation-based study, the annual cost associated with the care of DFU only in hospital admissions was calculated at \$US264 million/country ${ }^{(4)}$. These figures indicate that a substantial financial burden is currently present on the healthcare system that requires addressing with a multidisciplinary approach in DFU care.

Hyperglycaemia in diabetes stimulates the production of oxidative stress agents, which cause immunity dysfunction and a low-grade inflammatory state ${ }^{(5,6)}$. They underlie later macrovascular complications of diabetes including CHD, unstable angina and myocardial infarction ${ }^{(7)}$ and microvascular peripheral neuropathies ${ }^{(8)}$, co-morbidities associated with a worse prognosis in $\mathrm{DFU}^{(9)}$. Pro-inflammatory and immunecompromised states are implicated in the pathogenesis of DFU and are associated with chronic ulcers and lower extremity amputations ${ }^{(10)}$. It is conceivable that inflammation is a prelude to the development of peripheral neuropathy in the early-stage DFU. Disturbances of the immune system then interfere to exacerbate tissue homoeostasis as they turn primary DFU into chronic non-healing ulcers ${ }^{(11)}$. This importance has been the main incentive in recent years to evaluate mediators of inflammation and immunity in DFU and to assess their correlations with disease activity.

Up-regulation of chemokines C-C motif ligand 2/monocyte chemoattractant protein-1 (MCP-1) and IL-8 during wound repair causes abnormal leucocyte infiltration into the ulcerative tissue to facilitate bacterial colonisation at the sites of injury. IL-8 predominantly attracts neutrophils, and MCP-1 is a potent chemoattractant and activator of monocytes/macrophages ${ }^{(12)}$. Increased circulatory concentrations of MCP-1 as well as in the atherosclerotic plaques activate monocytes/macrophages to

Abbreviations: 25(OH)D, 25-hydroxyvitamin D; DFU, diabetic foot ulcers; hs-CRP, highly sensitive C-reactive protein; MCP-1, monocyte chemoattractant protein-1; ROC, receiver operating characteristic.

* Corresponding author: Professor A. Esteghamati, fax +98 216443 2466, email esteghamati@tums.ac.ir

$\dagger$ Both authors contributed equally to this work and their names are listed in alphabetical order. 
express the matrix metalloproteinases enzyme family ${ }^{(13)}$, tissue factor and superoxide anions ${ }^{(14,15)}$ aimed at degrading the protective fibrous cap of the atherosclerotic plaques to promote severe plaque instability. The subsequent cascade of events has a fundamental role in the initiation and progression of $\mathrm{CHD}^{(16)}$.

25-Hydroxyvitamin D $(25(\mathrm{OH}) \mathrm{D})$ is a major non-specific regulator of the immune system, which enhances the antimicrobial properties of monocytes/macrophages. Its receptor (vitamin D receptor; VDR) is conclusively found on the surface of almost all immune cells, including activated CD4+ and CD8+ T cells, B cells, neutrophils and antigen-presenting cells such as macrophages. 25(OH)D lowers pro-inflammatory cytokines while increasing anti-inflammatory response (e.g. inducing IL-10) ${ }^{(17)}$. As DFU reflect an immune-compromised state, many believe that defective systemic $25(\mathrm{OH}) \mathrm{D}$ production is more common and severe, especially in chronic nonhealing DFU.

Thus, we designed the present cross-sectional study with three main objectives: (i) to compare the serum concentrations of selected cytokines of the inflammatory system and 25(OH)D across the three groups, patients with chronic active DFU, control patients with type 2 diabetes and without DFU, and healthy controls; (ii) to assess the impact of serum $25(\mathrm{OH}) \mathrm{D}$ on the level of selected pro-inflammatory cytokines and their correlation with DFU; and (iii) to explore the inter-correlation of inflammatory markers and $25(\mathrm{OH}) \mathrm{D}$ and their association with various anthropometric and laboratory measurements of the study.

\section{Methods}

\section{Study design and patient recruitment}

Systematic subject recruitment was launched in January 2014 and ended in September 2014.

A hospital-based, cross-sectional design was adopted with sixty patients with type 2 diabetes and thirty healthy control subjects. Patients with diabetes were divided into those with chronic DFU who were admitted from their scheduled follow-up visits in the diabetes clinic of Vali-Asr Hospital (a teaching hospital affiliated with Tehran University of Medical Sciences (TUMS)) and those without a history of foot ulcers from our outpatient unit. The remaining individuals comprised healthy individuals of nearly the same age and with similar sexual distribution as the patients with diabetes. Patients were included consecutively if they were willing to participate and were diagnosed with type 2 diabetes as per the American Diabetes Association criteria( ${ }^{(18)}$. Exclusion criteria included type 1 diabetes, class III/IV heart failure according to the New York Heart Association, inflammatory or infectious diseases, autoimmune and rheumatic diseases, malignancy, haematological diseases, pregnancy, severe renal or liver failure/ cirrhosis, history of surgical and angioplasty interventions and treatment with anti-inflammatory drugs, immunosuppressants and vitamin D supplements. Patients in the control group did not display any clinical signs of infection. DFU was defined as a full-thickness skin defect that required at least 2 weeks for healing ${ }^{(19)}$. Our patient sample was selected from patients with grade II active DFU at the time of hospitalisation according to the University of Texas Wound Classification System as defined by grade I (superficial wound, not involving, tendon, capsule or bone), grade II (wound penetrating to tendon or capsule) and grade III (wound penetrating bone or joint). Grade 0 patients (pre- or post-ulcerative site that had healed) were also excluded from the study.

All procedures dealing with human rights were conducted in accordance with the guidelines laid down in the most recent revision of the Helsinki declaration. Written informed consents were obtained from the recruited participants before to enrolment, and the ethics committee of the TUMS approved the study protocol.

\section{Sampling and measurements}

Weight and height of the participants were determined in light clothing and without shoes. Waist circumference (WC) was calculated at mid-distance between the iliac crest and the rib cage and was rounded off to the nearest $0 \cdot 1 \mathrm{~cm}$. BMI was computed as weight $(\mathrm{kg})$ divided by height squared $\left(\mathrm{m}^{2}\right)$. The participants were instructed to rest for at least $5 \mathrm{~min}$ before having their blood pressure (BP) measured three times with at least 5-min intervals. BP was measured using a standard mercury sphygmomanometer (Baumanometer; W.A. Baum Co. Inc.) in a seated position and after 5 min of rest. Systolic (SBP) and diastolic (DBP) BP were recorded and the average values were considered for further analyses. Physicians with substantial experience (A. E. and M. N.) interviewed the participants and recorded the anthropometric measurements. At the time of measurement, investigators were blinded to the results of the biochemical measurements.

A volume of 10-ml venous blood samples were collected from each participant following a 12 -h overnight fast. Fasting plasma glucose (FPG) was measured by the glucose oxidase method. Proportion of glycated $\mathrm{Hb}$ (HbA1c) was determined using the HPLC (Skylight Biotech Inc.) method, and is expressed in both Diabetes Control and Complications Trial-derived (\%) and Information Sciences Institute (ISI) International Federation of Clinical Chemistry and Laboratory Medicine (IFCC)-recommended ( $\mathrm{mmol} / \mathrm{mol})$ units. Intra-assay and interassay CV were $2 \cdot 1$ and $2 \cdot 6 \%$, respectively, for glucose measurements. Concentration of total cholesterols (Chol), TAG, HDL-cholesterol and LDL-cholesterol were measured using enzymatic methods (Pars Azmoon commercial kits). The laboratory investigator was blinded to the anthropometric measurement results, physical examination and other medical records of the participants.

\section{Measurement of cytokines of inflammation and 25-hydroxyvitamin D}

Serum levels of MCP-1 were determined according to the manufacturer's instructions (Human MCP-1/MCAF ELISA kit catalog no. CSB-E04655h; Cusabio Biotech). The analytical range of detection was $31 \cdot 25-2000 \mathrm{pg} / \mathrm{ml}$, with a sensitivity of $30 \cdot 184 \mathrm{pg} / \mathrm{ml}$, and the intra-assay and inter-assay CV were 
$<8$ and $<10 \%$, respectively. For detecting serum IL-6 and IL-8 concentrations, human ELISA kits (sensitivity $<2 \mathrm{pg} / \mathrm{ml}$, range of detection: $62 \cdot 5-200 \mathrm{pg} / \mathrm{ml}$, incubation time: $105 \mathrm{~min}$ for IL-6 ELISA kit; sensitivity $<25 \mathrm{pg} / \mathrm{ml}$, range of detection: 62.5-2000 pg/ml, incubation time: $100 \mathrm{~min}$ for IL-8 ELISA kit) were used according to the proprietary kit protocol (catalog nos 950.030.048, 950.030.096, 950.030.192 (IL-6 ELISA kit) 950.050.048, 950.050.096, 950.050.192 (IL-8 ELISA kit); Diaclone). The intra-assay and inter-assay $\mathrm{CV}$ were 4.2 and $7.7 \%$ for the IL- 6 ELISA kit and 3.1 and $9.7 \%$ for the IL-8 ELISA kit, respectively. There was no cross-reactivity between the IL- 6 and IL-8 ELISA kits with other human cytokines. Serum 25(OH)D was measured using a competitive ELISA immunoassay kit (25(OH)D total ELISA, catalog no. 141917, incubation time: 120/30/15 min; DIAsource ImmunoAssays SA). The assay precision was characterised by intra-assay and inter-assay $\mathrm{CV}$ of $2.5-7.8$ and $4.3-9.2 \%$, respectively, with sensitivity of $2.8 \mathrm{ng} / \mathrm{ml}$, and a detection range of $0-180 \mathrm{ng} / \mathrm{ml}$. Serum concentrations of highly sensitive C-reactive protein (hs-CRP) were determined quantitatively using the ELISA method (Diagnostics Biochem Canada Inc.; catalog no. CAN-CRP-132360; kit size: ninety-six wells; kit type: e Sandwich ELISA; sensitivity: $10 \mathrm{ng} / \mathrm{ml}$; sample type/sample volume: human serum $/ 20 \mu \mathrm{l}$; calibrator range: 100-10 $000 \mathrm{ng} / \mathrm{ml}$; total incubation time: $55 \mathrm{~min}$ ).

\section{Sample size determination}

A priori power analysis using data from previous research involving $\mathrm{DFU}^{(13)}$ was conducted to determine the required number of participants $(n)$. For this purpose, an estimated effect size was calculated based on Cohen's $d^{(20)}$ of $2 \cdot 01$ for detecting the mean plasma MCP-1 difference per 1 sD. Subsequently, study sample size was calculated using the equations from Whitley \& Ball ${ }^{(21)}$. These calculations (online Supplementary Fig. S1) showed that each group required eleven participants to meet the required power of $99 \%$ at the $\alpha$ value $\leq 0 \cdot 05$. Therefore, the power of the study with thirty participants for each group was calculated to be $>99 \%$ for a type 1 error of 0.05 . Before study completion, two subjects from the healthy control group opted out of the study, whose data did not enter the final pool for analysis.

\section{Statistical methodology}

Data were analysed using Stata release 13 (StataCorp LP) and PASW software, version 18.0 (IBM Corp.). The Shapiro-Wilk test was used to evaluate the normality assumption. Outcome data are given as proportions (\%) for categorical variables, as mean values and standard deviations for continuous variables with Gaussian distribution, and as medians and interquartile ranges for continuous variables with non-Gaussian distribution. Categorical variables were compared using Fisher's exact $/ \chi^{2}$ tests. Cochran-Armitage test-for-trend was used to compare proportional ratios of each serum $25(\mathrm{OH}) \mathrm{D}$ tertile across the three groups of study. Continuous variables were compared in two groups using Student's $t$ test or Mann-Whitney $U$ test, as appropriate. Levene's $F$ was used to determine equality of variance between groups at $P>0 \cdot 05$. If the equality of variances was violated, the ancillary Welch's robust statistic was used to test the equality of means. Data that met the required assumptions were subject to one-way ANOVA. Significant results from ANOVA were further analysed using Bonferroni post boc test. The Kruskal-Wallis test was used as the non-parametric alternative when data did not meet each of the assumptions for ANOVA. $P_{\text {for trend }}$ values for serum MCP-1, IL-8 and hs-CRP among the tertiles of $25(\mathrm{OH}) \mathrm{D}$ were derived from the non-parametric Jonckheere-Terpstra test. Correlations of the cytokines of inflammation and $25(\mathrm{OH}) \mathrm{D}$ with the clinical and biochemical outcomes were tested using non-parametric Spearman's correlation coefficients $(r)$ with further Bonferroni adjustment for multiple comparisons. Receiver operating characteristic (ROC) curves were constructed to analyse the discriminative power of circulating levels of MCP-1, IL-6, IL-8, 25(OH)D and hs-CRP for the detection of DFU in patients with diabetes and with or without ulcers. Crude and two levels of multivariable adjusted logistic regression models were used to assess whether inflammatory cytokines and 25(OH)D are independently associated with the presence of DFU (binary outcome). In each model, OR and 95\% CI were calculated per one unit increment in the value of the circulating markers. At each step, collinearity diagnostics was run by exploring the tolerance and the variance inflation factor (VIF) in a criterion that is accompanied by examining the standard errors in each predictive model. In this definition, VIF $>10$ /tolerance $\leq 0 \cdot 1$, condition index $>30$ and variance proportions $>50 \%$ are carefully scrutinised for the multicollinearity, allowing the appropriate covariates to enter the models with $\mathrm{SE}$ values $<2$ for each independent variable. A two-tailed $P$ value of $<0.05$ was considered to be statistically significant.

\section{Results}

Baseline characteristics of the study participants are summarised in Table 1. Patients with DFU and control patients with diabetes and healthy controls had comparable age, sexual distribution, TAG, LDL-cholesterol, DBP and HbA1c. Female: male ratio, parameters of body composition (WC and BMI), Chol, HDL-cholesterol, SBP and FPG were significantly different between non-ulcerative patients with diabetes and patients with the diabetic foot. Although the trend of changes from healthy controls towards patients with DFU showed significant increases for serum MCP-1, 25(OH)D, IL-6 and hs-CRP and a decrease for IL-8, only serum MCP-1, IL-6 and hs-CRP were significantly different between DFU and non-DFU diabetes groups ( $P$ value $<0.001$ for serum MCP-1 and IL-6, $P$ value $=0.003$ for hs-CRP, respectively; Table 1 ).

From the results of bivariate correlations (Table 2), age, WC and BMI were correlated with the levels of inflammatory cytokines MCP-1, IL-6, hs-CRP and 25(OH)D; however, none was associated with serum IL- 8 concentration. Of note, levels of each pro-inflammatory cytokine and 25(OH)D correlated with raised FPG concentration and a poor glycaemic control (i.e. elevated HbA1c values; Table 2).

To explore the associations of the evaluated cytokines and $25(\mathrm{OH}) \mathrm{D}$, scatter plots of the correlations involving serum 
Table 1. Baseline characteristics of the study subjects

(Medians (variables with non-normal distribution) and interquartile ranges (IQR))

\begin{tabular}{|c|c|c|c|c|c|c|c|c|}
\hline & \multicolumn{2}{|c|}{ Healthy controls ( $n$ 28) } & \multicolumn{2}{|c|}{ T2DM controls $(n$ 30) } & \multicolumn{2}{|c|}{ DFU $(n 30)$} & \multirow[b]{2}{*}{$P^{\star}$} & \multirow[b]{2}{*}{$P$ for DFU $v$. T2DM controls } \\
\hline & Median & IQR & Median & IQR & Median & IQR & & \\
\hline MCP-1 (pg/ml) & 264.5 & 70.5 & 431.5 & $119 \cdot 2$ & $612 \cdot 0$ & $110 \cdot 0$ & $<0.001$ & $<0.001$ \\
\hline IL-6 (pg/ml) & 2.5 & $1 \cdot 6$ & 6.5 & $3 \cdot 1$ & 19.7 & $33 \cdot 2$ & $<0.001$ & $<0.001$ \\
\hline IL-8 (pg/ml) & 147.5 & $307 \cdot 7$ & 60.5 & $55 \cdot 0$ & $78 \cdot 0$ & 33.7 & 0.049 & 0.188 \\
\hline 25(OH)D (ng/ml) & 8.0 & 7.9 & $16 \cdot 0$ & $14 \cdot 1$ & $16 \cdot 8$ & $24 \cdot 6$ & 0.002 & 0.478 \\
\hline hs-CRP (ng/ml) & 6.35 & 6.7 & 31.5 & 29.5 & $47 \cdot 0$ & $26 \cdot 5$ & $<0.001$ & 0.003 \\
\hline Age (years) & $38 \cdot 0$ & 10.5 & 54.5 & 18.5 & $59 \cdot 0$ & $18 \cdot 2$ & $<0.001$ & 0.155 \\
\hline Sex ( $n$ female $/ n$ male $)$ & \multicolumn{2}{|c|}{$13 / 15$} & \multicolumn{2}{|c|}{$17 / 13$} & \multicolumn{2}{|c|}{$8 / 22$} & 0.018 & 0.058 \\
\hline $\mathrm{WC}(\mathrm{cm}) \dagger$ & $84 \cdot 0$ & $7 \cdot 3$ & $90 \cdot 2$ & 8.0 & 99.8 & $7 \cdot 0$ & $<0.001$ & $<0.001$ \\
\hline BMI $\left(\mathrm{kg} / \mathrm{m}^{2}\right)$ & 24.9 & $2 \cdot 7$ & $26 \cdot 4$ & 4.5 & 28.9 & 1.7 & $<0.001$ & $<0.003$ \\
\hline TAG (mmol/l) & $1 \cdot 13$ & 0.64 & 1.39 & 0.72 & 1.63 & 0.86 & 0.005 & 0.139 \\
\hline Chol $(\mathrm{mmol} / \mathrm{l}) \dagger$ & 4.24 & 1.01 & 4.43 & 0.91 & $5 \cdot 23$ & 1.34 & 0.002 & 0.019 \\
\hline LDL-cholesterol (mmol/l) & 2.42 & 0.99 & $2 \cdot 84$ & 0.91 & $2 \cdot 72$ & 0.44 & 0.300 & 0.469 \\
\hline HDL-cholesterol $(\mathrm{mmol} / \mathrm{l})$ & 1.15 & 0.37 & 1.09 & 0.39 & 1.00 & 0.25 & 0.013 & 0.021 \\
\hline $\mathrm{SBP}(\mathrm{mmHg})$ & $120 \cdot 0$ & $10 \cdot 0$ & 127.5 & 26.5 & $145 \cdot 0$ & $26 \cdot 2$ & $<0.001$ & 0.002 \\
\hline $\mathrm{DBP}(\mathrm{mmHg})$ & $80 \cdot 0$ & 8.7 & $80 \cdot 0$ & $15 \cdot 2$ & $80 \cdot 0$ & $15 \cdot 0$ & 0.247 & 0.325 \\
\hline $\mathrm{FPG}(\mathrm{mmol} / \mathrm{l})$ & 4.83 & 0.68 & 8.44 & 2.64 & 11.32 & 3.91 & $<0.001$ & $<0.001$ \\
\hline $\mathrm{HbA} 1 \mathrm{c} \%(\mathrm{mmol} / \mathrm{mol})$ & 4.9 & 0.4 & 7.9 & 1.8 & 8.5 & $2 \cdot 2$ & $<0.001$ & 0.100 \\
\hline
\end{tabular}

T2DM, type 2 diabetes mellitus; DFU, diabetic foot ulcers; MCP-1, monocyte chemoattractant protein-1; 25(OH)D, 25-hydroxyvitamin D; hs-CRP, highly sensitive C-reactive protein;

WC, waist circumference; Chol, total cholesterol; SBP, systolic blood pressure; DBP, diastolic blood pressure; FPG, fasting plasma glucose; HbA1c, glycated Hb.

* $P$ value for mediators of immunity and inflammations are calculated using the non-parametric Jonckheere-Terpstra trend test.

† Mean and standard deviation (variables with normal distribution).

MCP-1, IL-6, IL-8, hs-CRP and 25(OH)D are depicted in the online Supplementary Fig. S2. As the matrix of inter-correlations between markers of inflammation and $25(\mathrm{OH}) \mathrm{D}$ was similar across the three study groups, we decided to only demonstrate the overall matrix of correlation

Fig. 1 summarises the results of the ROC analysis of measured serum cytokines and $25(\mathrm{OH}) \mathrm{D}$ for the detection of DFU. In this figure, discriminatory power of included markers of inflammation and 25(OH)D for the diagnosis of diabetic foot among non-DFU patients with diabetes was evaluated in a multipleclassifier ROC plot. Serum IL-6 had the highest discriminatory power for the detection of DFU (area under the ROC curve (AUROC) $0.906 ; 95 \%$ CI 0.827, 0.984), the difference of which was statistically significant from the other biomarkers ( $P$ value $<0 \cdot 001$ ), followed by serum MCP-1 (AUROC 0.823; $95 \%$ CI $0.697,0.948$ ) and hs-CRP (AUROC 0.721; $95 \%$ CI 0.588, 0.854).

On the basis of the results of univariate and bivariate analyses, associations of inflammatory cytokines and $25(\mathrm{OH}) \mathrm{D}$ with DFU were explored in three models of the logistic regression analysis. In the crude logistic regression model, MCP-1, 25(OH)D and hs-CRP emerged as the strongest correlates of DFU (Table 3). Introduction of age, sex and BMI as clinically significant covariates in the primary adjusted model saw the rise of $25(\mathrm{OH}) \mathrm{D}$ concentrations as the most significant correlate of DFU $(P=0 \cdot 016$; OR $2 \cdot 476$; $95 \%$ CI $1 \cdot 188,5 \cdot 159)$ in a model with hs-CRP and MCP-1 as the other independent correlates for the occurrence of DFU. Despite additional adjustments for baseline confounders (HDL-cholesterol and HBA1c), serum 25(OH)D remained an independent correlate of DFU in the final adjusted model (OR 2.194; $95 \%$ CI 1.003, 4.415; Table 3).

Fig. 2 displays the impact of serum $25(\mathrm{OH})$ clustering on the levels of serum inflammatory cytokines and their OR for the occurrence of DFU. Stratification of the study population within the tertiles of $25(\mathrm{OH}) \mathrm{D}$ resulted in a progressively increased risk of belonging to the DFU subgroup for higher serum IL-8 concentration (Fig. 2).

On the basis of the impact of serum $25(\mathrm{OH}) \mathrm{D}$ clustering on the increased probability for the occurrence of DFU, we sought to determine the proportion of individuals within the tertiles of serum 25(OH)D across different subgroups of our study. As the online Supplementary Fig. S3 demonstrates, the upper tertile of serum $25(\mathrm{OH}) \mathrm{D}$ classifies a higher proportion of individuals with DFU and a lower percentage from the control groups ( $P_{\text {for }}$ trend value $=0.002$, online Supplementary Fig. S3).

\section{Conclusions}

Core findings. Conclusions drawn based on the main findings are as follows: (1) consistent increases in serum $25(\mathrm{OH}) \mathrm{D}$, MCP-1, IL-6 and hs-CRP from the disease-free state to active DFU, although the increase in circulating $25(\mathrm{OH}) \mathrm{D}$ levels from control patients with non-ulcerative diabetes to patients with diabetic foot did not reach the required level of significance; (2) non-linear alteration of serum IL-8 with an overall downward trajectory from healthy controls to patients with DFU, a finding that may suggest additional mechanisms involved in DFU target various inflammatory signal biomolecules differently; and (3) positive correlation of circulating 25(OH)D with DFU in the final adjusted model (OR 2.194; $95 \%$ CI 1.003, 4.415), higher levels of which progressively increase the risk of DFU due to pro-inflammatory cytokines across the strata of $25(\mathrm{OH}) \mathrm{D}$ status. We believe that the significant associations of MCP-1, IL-6, IL-8, hs-CRP and $25(\mathrm{OH}) \mathrm{D}$ with various clinical parameters including body composition indices, lipid profile, poor glycaemic control and BP readings are directly related to the higher risk for 


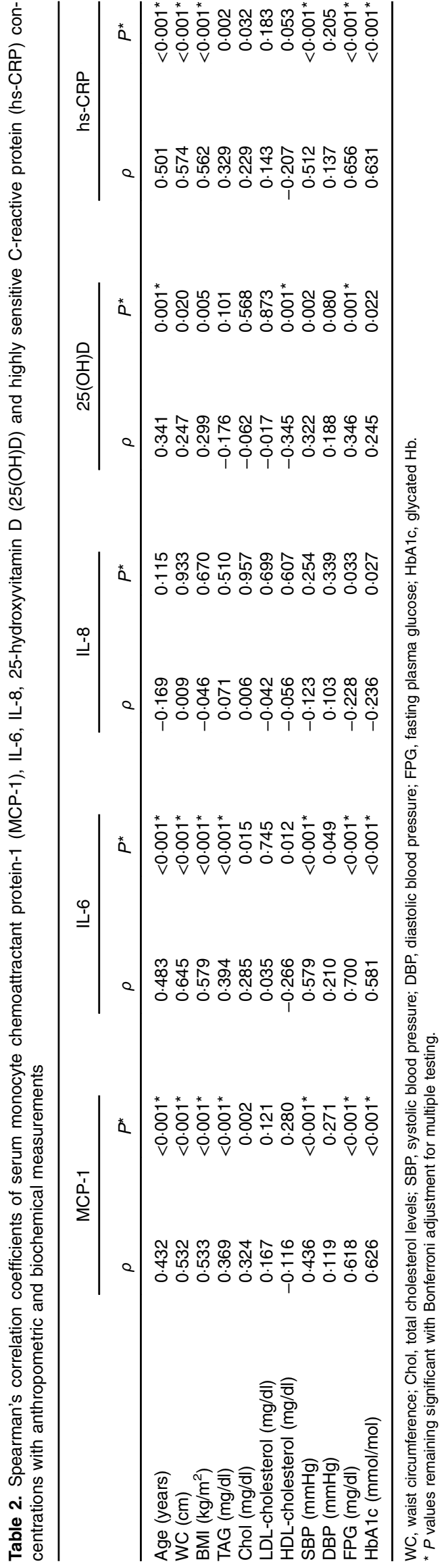

DFU among subjects with a modified inflammatory and $25(\mathrm{OH}) \mathrm{D}$ profile.

Independent associations of pro-inflammatory cytokines with diabetic foot ulcers. Raised serum levels of MCP-1 ${ }^{(10,13)}$ and hs-CRP (along with its inducer, IL-6) ${ }^{(11,13,22)}$ among our patients with DFU confirm the result of previous surveys, with the failure of chronic DFU to heal properly in time contemplated as the main reason for such observations ${ }^{(10)}$. Hs-CRP, in particular, is a new-found marker of diagnosis and follow-up of osteomyelitis ${ }^{(23)}$ and an independent predictor of progression from DFU to limb amputation in a number of prospective cohorts ${ }^{(24,25)}$.

To the best of our knowledge, the present study is the first to underscore a positive correlation between the circulating levels of $25(\mathrm{OH}) \mathrm{D}$ and the active chronic DFU. Although we could not locate a ROC curve with satisfying AUROC readings, serum $25(\mathrm{OH}) \mathrm{D}$ was a major determinant of DFU in the crude and multivariable logistic regressions (more than 2-fold increased risk in the final adjusted model). This is separately confirmed by the finding that OR of serum IL-8 for the occurrence of DFU significantly increases over the higher strata of serum $25(\mathrm{OH}) \mathrm{D}$. The mean serum 25(OH)D in patients with DFU $(26.5 \mathrm{ng} / \mathrm{ml})$ lies halfway between the cut-off values by the definition of two of the most trusted clinical guidelines for vitamin D deficiency prevention and treatment $(\geq 20 \mathrm{ng} / \mathrm{ml}$ by the Institute of Medicine ${ }^{(26)}$ and $\geq 30 \mathrm{ng} / \mathrm{ml}$ by The Endocrine Society $\left.{ }^{(27)}\right)$. These two cut-off values have been recommended as thresholds for adequate serum 25(OH)D concentrations for bone density health outcomes. However, for extraskeletal outcomes of cancer, CVD, diabetes and autoimmune disorders, there is a lack of consistent, conclusive and sufficient evidence to support a causality effect scheme and help establish the measurement definition criteria by informing the nutritional intake requirements ${ }^{(26,27)}$.

Contrary to our findings, recent reports involving DFU observed markedly higher rates of 25(OH)D insufficiency and deficiency among diabetic foot patients compared with their ulcer-free counterparts with diabetes and healthy controls $^{(22,28,29)}$. Considering the role of $25(\mathrm{OH}) \mathrm{D}$ to provoke insulin sensitivity and secretory transcription factors within seconds of impact on its receptor VDR (present on the surface of pancreatic islets cells, adipocytes and peripheral skeletal muscle cells), 25(OH)D deficiency has been suggested in the pathogenesis of DFU due to an inefficient removal of excessive glucose from the circulation ${ }^{(28)}$. Moreover, the inhibitory and stimulatory mechanisms of $25(\mathrm{OH}) \mathrm{D}$ on the osteoprotegerin and receptor activator of NF-KB ligand (RANKL) ligand systems, respectively, have been associated with the correlation between lower serum 25(OH)D concentrations and the severity of vascular calcifications in $\mathrm{DFU}^{(30)}$.

$25(\mathrm{OH}) \mathrm{D}$ is increasingly being recognised as a non-specific regulator of immunity and may have a preventive role in the pathogenesis of diabetic foot and joint infection by providing an enhanced level of antimicrobial protection ${ }^{(31)}$. However, various parameters of immunity including $25(\mathrm{OH}) \mathrm{D}$ may remain intact or even increase in diabetes and diabetic foot 


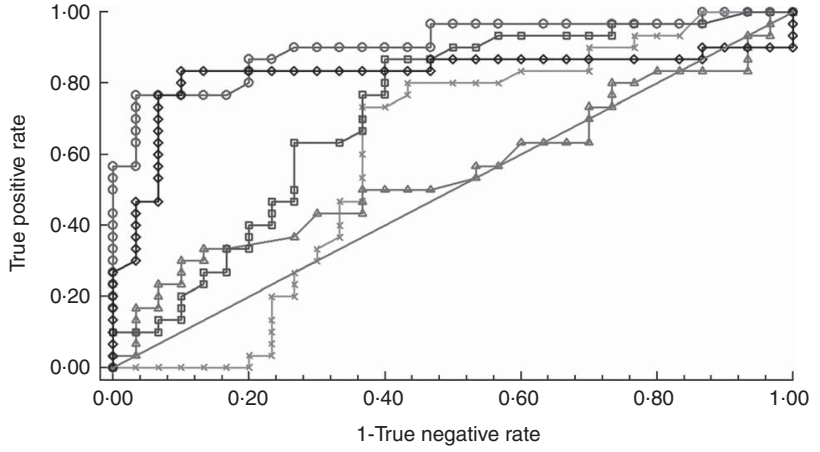

Fig. 1. Non-parametric receiver operating characteristic (ROC) curve demonstrating the discriminatory power of the serum cytokines of inflammation and 25-hydroxyvitamin $D(25(\mathrm{OH}) \mathrm{D})$ to detect patients with active diabetic foot ulcer (DFU) among the subset with diabetes. Results of the ROC analysis confirm serum IL- 6 as the factor with the highest discriminatory power for the detection of DFU. $P$ value of the difference between ROC curves was statistically significant $(P<0.001) .-$, Serum IL-6 (area under the ROC curve (AUROC) $0.907 \pm 0.040 ; 95 \% \mathrm{Cl} 0.827,0.984) ;-\triangle$, serum $25(\mathrm{OH}) \mathrm{D}$ (AUROC $0.553 \pm 0.076 ; 95 \% \mathrm{Cl} 0.404,0.703$ ); $\diamond$, serum monocyte chemoattractant protein-1 (AUROC 0.823 $\pm 0.064 ; 95 \% \mathrm{Cl} 0.697,0.948$ ); $\times$, serum IL-8 (AUROC 0.599 $\pm 0.079 ; 95 \% \mathrm{Cl} 0.444,0.754) ; \square$, serum highly sensitive C-reactive protein (AUROC $0.721 \pm 0.068 ; 95 \% \mathrm{Cl} 0.588$, 0.854).

to reflect an 'altered immunity' status rather than the widely accepted 'immunodeficiency' concept ${ }^{(32)}$. This altered immunophenotype was elucidated in an elegant cytokine study of diabetes patients with or without foot ulcers. Among other findings, specific-type alterations in the regulators of immunity and inflammation were observed, including an upsurge in hs-CRP levels, a down-regulation of RANTES (regulated on activation, normal $\mathrm{T}$ cell expressed and secreted) coupled with sera IL-8 and MCP-1 remaining constant ${ }^{(11)}$. Other scenarios may include a ubiquitous lack of $25(\mathrm{OH}) \mathrm{D}$ action due to actionresistance and the subsequent rise of serum $25(\mathrm{OH}) \mathrm{D}$, similar to that of metabolic hormones (e.g. leptin) in chronic states of low-grade inflammation and hyperinsulinaemia as present in diabetes and its complications.

Another possibility to explain the rise of serum $25(\mathrm{OH}) \mathrm{D}$ in DFU concerns the strong negative correlation of circulating $25(\mathrm{OH}) \mathrm{D}$ with IL-8 in each subset of our recruited individuals (in the total study population, $P$ value $=0.007$ and Spearman's $\rho$ correlation coefficient $=-0 \cdot 286$ ). This association in the subset of patients with diabetic ulcers is especially noteworthy given the concurrent unusual increase and decrease in the overall serum $25(\mathrm{OH}) \mathrm{D}$ and IL-8 concentrations, respectively. Interestingly, Tiwari et al. ${ }^{(22)}$ also pointed towards such a negative correlation of $25(\mathrm{OH}) \mathrm{D}$ and cytokines in diabetic foot individuals, although using a different set of cytokines from that of our study - namely, IL- 6 , IL- $1 \beta$ and TNF- $\alpha$. We speculate that escalating $25(\mathrm{OH}) \mathrm{D}$ levels may in turn represent a salvage counter-regulatory mechanism against the systemic pro-inflammatory status present in DFU (i.e. as derived by the chemoattractant IL-8 among other inflammatory biomarkers), a hypothesis supported by a low up-regulation of IL-8 in DFU tissues compared with other specimen immuno-inflammatory biomarkers ${ }^{(12)}$. Hence, the lack of statistically significant difference found in serum IL- 8 between patients with diabetes

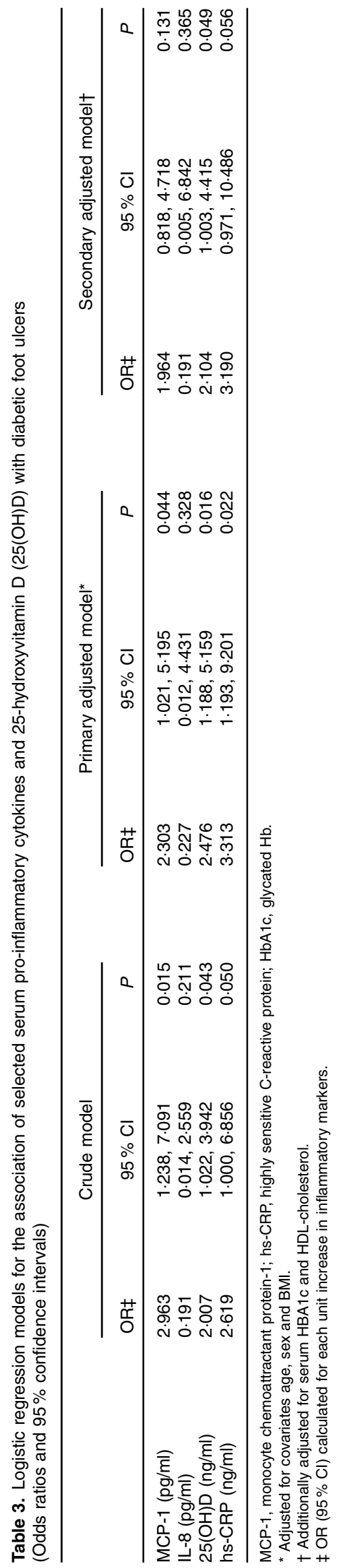




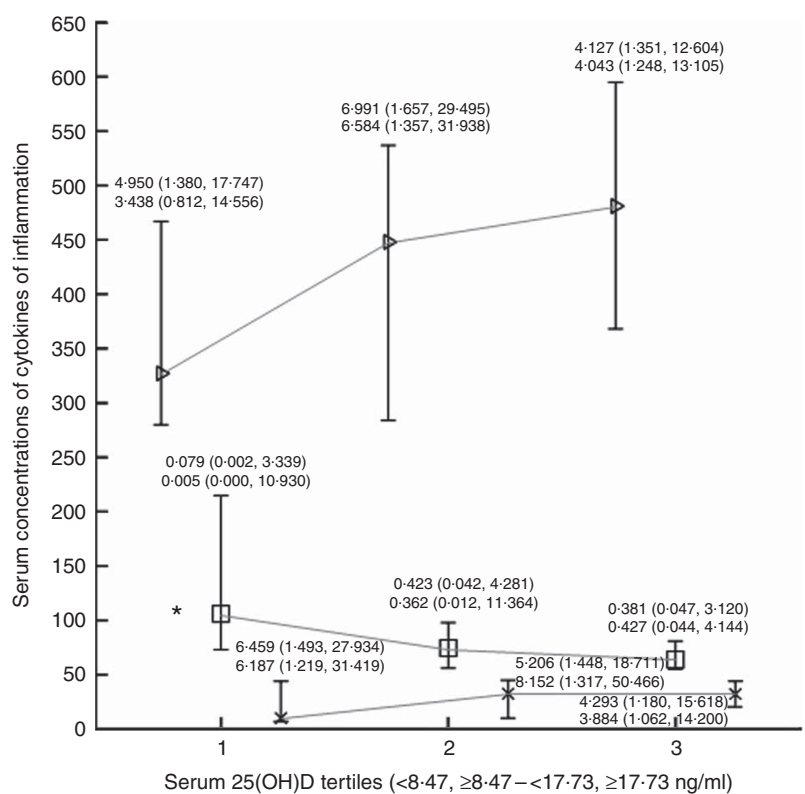

Fig. 2. Association of selected cytokines of inflammation with diabetic foot ulcer (DFU) within the tertiles of serum 25-hydroxyvitamin D (25(OH)D). $P$ values denoting the difference in serum concentrations of inflammatory cytokines between the first and the third tertiles of serum 25(OH)D are statistically non-significant for monocyte chemoattractant protein-1 (MCP-1) and highly sensitive C-reactive protein (hs-CRP) and significant for serum IL-8 values $\left(P_{\text {for }}\right.$ trend values: $0.066,0.005,0.249$; for MCP-1, IL-8 and hs-CRP, respectively). OR and $95 \% \mathrm{Cl}$ for the occurrence of DFU by the effect of fitting into different serum 25(OH)D tertiles are shown next to each error bar (upper row denotes unadjusted and lower row denotes adjusted for age, sex and BMI). Values are medians, with $95 \% \mathrm{Cl}$ represented by error bars. ${ }^{*} P=0.005$. $\triangleright$, Serum MCP-1 (pg/ml); $\square$, serum IL-8 (pg/ml); X, serum hs-CRP (ng/ml).

with and without ulcers could be a consequence of the relatively unaffected levels of serum IL-8 by the diabetic ulcer conditions and contrary to other pro-inflammatory cytokines. In line with our suggestion, McInnes et al. ${ }^{(33)}$ recently identified diverse bidirectional alterations in the levels of pro-inflammatory cytokines, growth factors and receptors from the analysis of wound fluid samples of patients with DFU.

$25(\mathrm{OH}) \mathrm{D}$ reduces inflammatory response by inhibiting IL-8 production from rhinovirus-affected airway epithelial cells ${ }^{(34)}$. Targeting the link between $25(\mathrm{OH}) \mathrm{D}$ and IL-8 synthesis has revealed RhoA/Rho kinase and NF- $\kappa \mathrm{B}$ pathways as the primary routes for IL-8-reducing effects of elocalcitol (a VDR agonist) in benign prostatic hyperplasia ${ }^{(35)}$. Recently, it was shown 1,25-dihydroxyvitamin $\mathrm{D}$ supplementation is capable of suppressing both IL- 8 and MCP-1 in high-glucose-treated U937 monocytes ${ }^{(36)}$. However, IL-8-decreasing actions of $25(\mathrm{OH}) \mathrm{D}$ may also serve as a double-edged sword, as it also potentially inhibits the potent and beneficial angiogenic properties of IL- 8 in DFU. Taken together, the gap in data concerning the rise of serum $25(\mathrm{OH}) \mathrm{D}$ and its impact on the release of IL-8 pro-inflammatory cytokine in diabetic foot requires prompt addressing in future histopathological studies.

Limitations and strengths. Our study has several limitations. Primarily, a cross-sectional design hinders the validity of assumption on the order of causality between the rise of serum inflammatory cytokines, $25(\mathrm{OH}) \mathrm{D}$ and the occurrence of DFU. Our healthy and diabetic controls were not pre-specified for matching with their DFU cases. Despite a high study power, a relatively low number of included subjects may have introduced a bias with the interpretation of study findings. Finally, diverse and additive mechanisms involved in the inflammatory response should ideally be looked upon with the assessment of additional cytokines in later studies involving DFU. The strength of our study includes concurrent investigation of selected pro-inflammatory cytokines and $25(\mathrm{OH}) \mathrm{D}$ in three groups of healthy individuals, non-ulcerative control patients with diabetes and diabetic foot by using a robust statistical methodology. To the best of our knowledge, the present study is the first to evaluate the association between a selection of pro-inflammatory cytokines and active DFU by stratifying the OR for each cytokine within the tertiles of serum 25(OH)D status.

In summary, we demonstrated the associations of selected serum pro-inflammatory cytokines and $25(\mathrm{OH}) \mathrm{D}$ with the chronic active DFU. In particular, 25(OH)D and IL-8 seem to share a common pathway in patients with the ulcerative diabetic foot, as reflected by their negative correlation in our study and the biological plausibility of their interaction in other bodily disorders; however, this needs to be further realised. Although we were unable to demonstrate a significant difference in serum $25(\mathrm{OH}) \mathrm{D}$ levels between control non-ulcerative patients with diabetes and patients with active DFU, the continuous rise of serum $25(\mathrm{OH})$ from healthy controls to patients with the active DFU is in sharp contrast to previous reports involving the assessment of serum 25(OH)D in DFU and should be followed-up by further well-designed investigations. We recommend prospective observational cohorts to evaluate the correlation between the baseline levels of serum $25(\mathrm{OH}) \mathrm{D}$ and the occurrence of incident DFU in patients with ulcer-free diabetes. Specifically, other groups are required to study the nature of the perplexing rise of serum $25(\mathrm{OH}) \mathrm{D}$ in $\mathrm{DFU}$ and determine whether this increase in circulating $25(\mathrm{OH})$ levels is secondary to the inflammatory derangement present in DFU as part of a complex counter-regulatory response, has a direct pathogenic role in the development or progression of DFU or reflects the altered function of the immune system present in DFU. At this stage and based on these concerns, we cannot comment on the clinical implication of vitamin $\mathrm{D}$ status on the prevention (i.e. as an alternative source for screening and/or prediction of DFU), treatment (e.g. as an adjunctive treatment focused on the reduction of serum $25(\mathrm{OH}) \mathrm{D}$ in DFU or non-ulcerative diabetes) or outcome of DFU. However, similar findings to our study may facilitate executing trial runs of cytokine-targeted therapy as an add-on therapy to the conventional diabetic foot debridement, pressure alleviating and antibiotic treatment for practising a stateof-art care of patients with DFU.

\section{Acknowledgements}

The authors wish to thank the patients and staff of Vali-Asr Hospital for their gracious participation in this study. A. E. takes full responsibility for the work as a whole, including the study 
design, access to data and the decision to submit and publish the manuscript.

This research received no specific grant from any funding agency, commercial or not-for-profit sectors.

M. A., P. G., M. N. and A. E. conceived the study, participated in its design, coordination and acquisition of data. A. E. and M. N. interpreted the findings, interviewed all participants and revised the manuscript critically. M. A., S. N. and A. G. wrote the manuscript and performed the statistical analysis. All authors read and approved the final manuscript

None of the authors has any conflicts of interest to declare.

\section{Supplementary material}

For supplementary material/s referred to in this article, please visit http://dx.doi.org/10.1017/S0007114516001094

\section{References}

1. Bakker K \& Schaper N (2012) The development of global consensus guidelines on the management and prevention of the diabetic foot 2011. Diabetes Metab Res Rev 28, $116-118$.

2. Lavery LA, Armstrong DG, Wunderlich RP, et al. (2003) Diabetic foot syndrome evaluating the prevalence and incidence of foot pathology in Mexican Americans and nonHispanic whites from a diabetes disease management cohort. Diabetes Care 26, 1435-1438.

3. Boulton AJ, Vileikyte L, Ragnarson-Tennvall G, et al. (2005) The global burden of diabetic foot disease. Lancet $\mathbf{3 6 6}$, 1719-1724.

4. Rezende K, Ferraz M, Malerbi D, et al. (2010) Predicted annual costs for inpatients with diabetes and foot ulcers in a developing country - a simulation of the current situation in Brazil. Diabet Med 27, 109-112.

5. Garcia C, Feve B, Ferre P, et al. (2010) Diabetes and inflammation: fundamental aspects and clinical implications. Diabetes Metab 36, 327-338.

6. Kalupahana NS, Moustaid-Moussa N \& Claycombe KJ (2012) Immunity as a link between obesity and insulin resistance. Mol Aspects Med 33, 26-34.

7. Brownlee M (2005) The pathobiology of diabetic complications a unifying mechanism. Diabetes 54, 1615-1625.

8. Dyck PJ \& Giannini C (1996) Pathologic alterations in the diabetic neuropathies of humans: a review. J Neuropathol Exp Neurol 55, 1181-1193.

9. Pinto A, Tuttolomondo A, Di Raimondo D, et al. (2008) Cardiovascular risk profile and morbidity in subjects affected by type 2 diabetes mellitus with and without diabetic foot. Metabolism 57, 676-682.

10. Dinh T, Tecilazich F, Kafanas A, et al. (2012) Mechanisms involved in the development and healing of diabetic foot ulceration. Diabetes 61, 2937-2947.

11. Weigelt C, Rose B, Poschen U, et al. (2009) Immune mediators in patients with acute diabetic foot syndrome. Diabetes Care 32, 1491-1496

12. Galkowska H, Wojewodzka U \& Olszewski WL (2006) Chemokines, cytokines, and growth factors in keratinocytes and dermal endothelial cells in the margin of chronic diabetic foot ulcers. Wound Repair Regen 14, 558-565.

13. Arya AK, Pokharia D, Bhan S, et al. (2012) Correlation between IL-7 and MCP-1 in diabetic chronic non healing ulcer patients at higher risk of coronary artery disease. Cytokine $\mathbf{6 0}$, 767-771.

14. Schecter AD, Rollins BJ, Zhang YJ, et al. (1997) Tissue factor is induced by monocyte chemoattractant protein-1 in human aortic smooth muscle and THP-1 cells. J Biol Chem 272, 28568-28573.

15. Ernofsson M \& Siegbahn A (1996) Platelet-derived growth factor-BB and monocyte chemotactic protein-1 induce human peripheral blood monocytes to express tissue factor. Thromb Res 83, 307-320.

16. Deo R, Khera A, McGuire DK, et al. (2004) Association among plasma levels of monocyte chemoattractant protein-1, traditional cardiovascular risk factors, and subclinical atherosclerosis. $J$ Am Coll Cardiol $\mathbf{4 4}$ $1812-1818$

17. Albar Z (2010) Vitamin D and inflammation. Indonesian I Rheumatol 4, 4-10.

18. American Diabetes Association (2013) Diagnosis and classification of diabetes mellitus. Diabetes Care 36, S67-S74.

19. Jeffcoate WJ, Game F \& Cavanagh PR (2005) The role of proinflammatory cytokines in the cause of neuropathic osteoarthropathy (acute Charcot foot) in diabetes. Lancet $\mathbf{3 6 6}$, 2058-2061.

20. Cohen J (1992) A power primer. Psychol Bull 112, 155-159.

21. Whitley E \& Ball J (2002) Statistics review 4: sample size calculations. Crit Care 6, 335-341.

22. Tiwari S, Pratyush DD, Gupta SK, et al. (2014) Vitamin D deficiency is associated with inflammatory cytokine concentrations in patients with diabetic foot infection. Br J Nutr 112, 1938-1943.

23. Michail M, Jude E, Liaskos C, et al. (2013) The performance of serum inflammatory markers for the diagnosis and follow-up of patients with osteomyelitis. Int J Low Extrem Wounds $\mathbf{1 2}$, 94-99.

24. Li X, Xiao T, Wang Y, et al. (2011) Incidence, risk factors for amputation among patients with diabetic foot ulcer in a Chinese tertiary hospital. Diabetes Res Clin Pract 93, 26-30.

25. Pickwell K, Siersma V, Kars M, et al. (2015) Predictors of lower-extremity amputation in patients with an infected diabetic foot ulcer. Diabetes Care 38, 852-857.

26. Ross AC, Manson JE, Abrams SA, et al. (2011) The 2011 report on dietary reference intakes for calcium and vitamin D from the Institute of Medicine: what clinicians need to know. J Clin Endocrinol Metab 96, 53-58.

27. Holick MF, Binkley NC, Bischoff-Ferrari HA, et al. (2011) Evaluation, treatment, and prevention of vitamin D deficiency: an Endocrine Society clinical practice guideline. J Clin Endocrinol Metab 96, 1911-1930.

28. Zubair M, Malik A, Meerza D, et al. (2013) 25-Hydroxyvitamin D [25 (OH) D] levels and diabetic foot ulcer: is there any relationship? Diabetes Metab Syndr 7, 148-153.

29. Tiwari S, Pratyush DD, Gupta B, et al. (2013) Prevalence and severity of vitamin D deficiency in patients with diabetic foot infection. BrJ Nutr 109, 99-102.

30. Swain J, Tiwari S, Pratyush D, et al. (2012) Vascular calcification in diabetic foot and its association with calcium homeostasis. Indian $J$ Endocrinol Metab 16, S450-S452.

31. Maier GS, Horas K, Seeger JB, et al. (2014) Is there an association between periprosthetic joint infection and low vitamin D levels? Int Orthop 38, 1499-1504.

32. Moazezi Z, Hosseinian A, Moazam EA, et al. (2013) Evaluation of immunological parameters in diabetic patients: are these patients immunodeficient? Iran J Allergy Asthma Immunol 13, 110-119. 
33. McInnes RL, Cullen BM, Hill KE, et al. (2014) Contrasting host immuno-inflammatory responses to bacterial challenge within venous and diabetic ulcers. Wound Repair Regen 22, 58-69.

34. Gross A, Sutherland E \& Chu H (2013) Vitamin D reduces inflammatory response to rhinovirus in human airway epithelium. Am J Respir Crit Care Med 187, A5968.

35. Penna G, Fibbi B, Amuchastegui S, et al. (2009) The vitamin D receptor agonist elocalcitol inhibits IL-8-dependent benign prostatic hyperplasia stromal cell proliferation and inflammatory response by targeting the RhoA/Rho kinase and NF-kB pathways. Prostate 69, 480-493.

36. Jain SK \& Micinski D (2013) Vitamin D upregulates glutamate cysteine ligase and glutathione reductase, and GSH formation, and decreases ROS and MCP-1 and IL- 8 secretion in highglucose exposed U937 monocytes. Biochem Biophys Res Commun 437, 7-11. 